\title{
Impact of hemodialysis on the prognosis of multiple myeloma: A nationwide population-based study and single-institute analysis
}

\author{
CHAO-FENG CHANG ${ }^{1}$, WU-CHIEN CHIEN ${ }^{2,3}$, CHI-HSIANG CHUNG ${ }^{3,4}$, JIH-CHIN LEE ${ }^{5}$, \\ SHUN-NENG HSU ${ }^{6}$ and JIA-HONG CHEN ${ }^{7,8}$
}

\begin{abstract}
${ }^{1}$ Department of Medicine, Division of Gastroenterology, and ${ }^{2}$ Department of Medical Research; ${ }^{3}$ School of Public Health, National Defense Medical Center; ${ }^{4}$ Taiwanese Injury Prevention and Safety Promotion Association; ${ }^{5}$ Department of Otolaryngology-Head and Neck Surgery; ${ }^{6}$ Department of Medicine, Division of Nephrology; ${ }^{7}$ Department of Medicine, Division of Hematology/Oncology, National Defense Medical Center, Tri-Service General Hospital, Taipei 11490;

${ }^{8}$ Graduate Institute of Clinical Medicine, College of Medicine, Taipei Medical University,
\end{abstract} Taipei 11031, Taiwan, R.O.C.

Received October 17, 2017; Accepted April 19, 2018

DOI: $10.3892 / 01.2018 .8857$

\begin{abstract}
Myeloma-associated kidney disease (MRKD) occurs in $\leq 40 \%$ patients with multiple myeloma (MM). The impact of hemodialysis (HD) on patients with MM was investigated. Between 2000 and 2010, a total of 1,610 patients in Taiwan were enrolled from the National Health Institute Research Database. MM was an independent risk factor for HD following adjustment via multivariate logistic regression analysis (adjusted hazard ratio, 7.347; 95\% confidence interval, 6.156-8.768; log-rank test, $\mathrm{P}<0.001)$. In addition, a notable decrease in survival rate was determined in patients with MM who underwent HD in the first year since diagnosis of MM. A total of $198(61.49 \%)$ patients received HD in the first year. Patients with MM with a lower frequency of HD in the first year had a relatively good prognosis. The present study confirmed that MM was a risk factor for HD. Significant early mortality in the first year was determined in patients with MM who underwent HD; however, renal recovery following therapeutic management was a prognostic factor. In addition to anti-myeloma therapy, early initiation of HD was beneficial following risk stratification of MRKD; however, an increased probability of recovery of renal function and discontinuation of dialysis, was demonstrated in patients with MM following $\mathrm{HD}$, compared with patients with MM without HD.
\end{abstract}

Correspondence to: Dr Jia-Hong Chen, Department of Medicine, Division of Hematology/Oncology, National Defense Medical Center, Tri-Service General Hospital, 325 Cheng-Kung Road, Taipei 11490, Taiwan, R.O.C.

E-mail:ndmc_tw.tw@yahoo.com.tw

Key words: multiple myeloma, myeloma-associated kidney disease, hemodialysis, renal recovery, prognosis, Taiwan

\section{Introduction}

Multiple myeloma (MM) is a malignant neoplasm involving plasma cell proliferation and overproduction of immunoglobulins (1). The incidence of MM is estimated to be $4 / 100,000$ people/year in the USA in 2004 (2) and 0.6/100,000 people/year in China in 2016 (3). MM is relatively uncommon and accounts for $1 \%$ all cancer types (4). MM is prevalent in elderly males and African-Americans $(1,5)$. The diagnostic criteria of MM include plasmacytosis of $>10 \%$ in the bone marrow and $\mathrm{M}$ protein in the urine or blood. Typical manifestations of myeloma-associated organ impairment include: Hypercalcemia, renal impairment, anemia and lytic bony lesions (4). Renal insufficiency caused by MM is termed myeloma-associated kidney disease (MRKD). MRKD is a common complication that occurs in 15-40\% patients with MM (1). The most common pathophysiology of MRKD is caused by myeloma cast nephropathy, which is an accumulation of light chain proteins due to overabundance that gives rise to tubulointerstitial fibrosis (6). Additionally, other predisposing factors, including hypercalcemia, volume depletion, hyper-viscosity, radiocontrast media use and nonsteroidal anti-inflammatory drugs (NSAIDs), are considered to be associated with MRKD (7).

The clinical strategies for patients with MM with MRKD include hydration, chemotherapy and emergency hemodialysis (HD), which attempt to rescue renal function. Only $1 \%$ patients with MM suffer irreversible renal failure requiring chronic HD followed by prompt treatment of the aforementioned predisposing factors (8). The average mean survival time following diagnosis with MM is 3 years, compared with $<2$ years for those with renal failure (7). Renal failure is considered a predictive factor for the outcome of MM, and renal recovery in patients with MRKD serves an important role during the disease course of MM. Clinically, HD is commonly used to manage acute kidney injury in MRKD (8). The aim of the present study was to determine the advantages of HD intervention and the prognosis of patients with MM. To the best 
of our knowledge, this is the first large-scale study to identify the risk of HD on patients with MM in Asia. The association between HD and the prognosis of patients with MM was also investigated. In addition, an analysis of a single institute regarding patients with $\mathrm{MM}$ with renal function impairment was conducted (Tri-Service General Hospital).

\section{Materials and methods}

Data source. The Taiwan National Health Insurance Research Database (NHIRD) was established in 1995, and The Taiwan National Health Insurance Administration Ministry of Health and Welfare (Taipei, Taiwan, R.O.C.) provide a number of medical services, including inpatient, outpatient and emergency services to $>99 \%$ of the entire population in Taiwan (9). In the present study, data from the NHIRD was used. The investigation protocols were approved by the official peer review committee in Tri-Service General Hospital (Taipei, Taiwan, R.O.C.). The diagnoses were made according to the International Classification of Diseases, 9th Revision, Clinical Modification (ICD-9-CM) (10). A single-institute analysis on the profiles of patients diagnosed with MM was conducted according to the diagnostic criteria of the International Myeloma Working Group (4). The patients were treated at the Tri-Service General Hospital (TSGH; Taipei, Taiwan) between January 1, 2002 and December 31, 2012. The patients' clinical information were extracted from medical records, and survival status was tracked by a case manager in the Cancer Registry Group of the TSGH. The present study was approved by the Institutional Review Board of the TSGH.

Patients and study design. A retrospective cohort design was used, and data was extracted from the NHIRD regarding patients between January 1, 2000 and December 31, 2010 in Taiwan. The inclusion criteria of MM in the case group were implemented according to ICD-9-CM 203.0. The exclusion criteria included: Diagnosis of MM made prior to 2000, renal failure or dialysis prior to tracking, age of $<20$ years and unknown sex. Patients without MM made up the control group. Notable variables included age, sex, and the comorbidities of diabetes mellitus (DM) (ICD-9-CM 250), hypertension (ICD-9-CM 401-405), chronic obstructive pulmonary disease (COPD) (ICD-9-CM 491-493, 406), coronary artery disease (CAD) (ICD-9-CM 410-414) and stroke (ICD-9-CM-430-428). There were 322 individuals in the case group and 1,288 individuals in the control group. At the end of the tracking period, December 31st 2010, 283 individuals remained alive in the case group and 354 individuals in the control group who had received HD (ICD-9-CM OP39.95). A total of 45 individuals $(13.9 \%)$ began HD in the first month after diagnosis with MM, and due to acute or subacute kidney injury caused by MM. The other patients progressed to end-stage renal disease and were treated with HD (Fig. 1). Furthermore, 283 patients with MM underwent HD were divided into treatment groups: HD alone (199 patients) and HD combined with anti-myeloma therapy (84 patients). The patients with MM with anti-myeloma therapy were treated with thalidomide, lenalidomide or bortezomib. Sub-analysis of cumulative overall survival rate between patients treated with HD and anti-myeloma therapy and patients treated with HD alone was conducted. Also, another sub-analysis for patients with MM stratified by bone marrow auto-transplantation was performed.

Secondly, a multiple clinical case study was conducted. The patients were enrolled between January 1, 2002 and December 31, 2012 at the TSGH with an initial diagnosis of MM, and there were 158 individuals, of which 19 had confirmed renal impairment. Using the Acute Kidney Injury Network (AKIN) criteria as a reference (11), a creatinine level $>4 \mathrm{mg} / \mathrm{dl}$ was used to define renal impairment. The clinical information collected from medical records included age, disease stage at diagnosis, creatinine level, estimated glomerular filtration rate (eGFR), AKIN criteria, frequency of emergency HD, dialysis dependence prior to diagnosis, plasma exchange following diagnosis with MM, renal biopsy, follow-up duration and survival status.

Statistical analysis. All analyses were conducted using SPSS software (version 18; SPSS, Inc., Chicago, IL, USA). The $\chi^{2}$ and Fisher's exact test was used for analysis of categorical variables, such as sex and comorbidities. The Student's t-test was used for continuous variable, such as age, and the data was presented as mean \pm standard error of the mean. Multivariate Cox regression was used to adjust the independent variables and to determine the association between each risk factor and HD. Hazard ratio (HR) and 95\% confidence interval (CI) were used to evaluate the relative risks between each risk factor. The Kaplan-Meier method was conducted to identify the cumulative incidence of HD and the log-rank test to determine the statistical significance between groups. All reported P-values were two-tailed, and $\mathrm{P}<0.001$ was considered to indicate a statistically significant difference. The cumulative survival curve of mortality for patients with MM with and without HD was constructed. Bar charts were used to investigate the association between HD and prognosis in patients with MM (horizontal axis, frequency of HD; vertical axis, number of patients with MM). The distribution of mortality/survival rate of the patients with MM is presented.

\section{Results}

The clinical characteristics of the patients from the present study are detailed in Table I. The distributions of sex, age, DM, hypertension, COPD, CAD and stroke between the two groups (with and without MM) were similar. The mean age was $68.12 \pm 13.29$ years in those with MM and $66.39 \pm 18.44$ in those without MM. Males outnumbered females in both groups at the end of follow-up (59.01\% vs. $40.99 \%)$ in the total population. A total of $283(87.89 \%)$ patients underwent HD in the case group and $354(27.48 \%)$ in the control group. Following adjustment of variables using the Charlson comorbidity index (CCI) (12), there was no statistical difference in clinical characteristics between the case and control groups. The Kaplan-Meier graph indicated that the requirement of HD was significantly higher in patients with MM than patients without MM (log-rank test $\mathrm{P}<0.001$; Fig. 2). As displayed in Table II, there was a significantly higher risk of HD in patients with MM than in those without MM [adjusted HR (aHR)=7.347; 95\% CI=6.156-8.768; $\mathrm{P}<0.001]$. The risk of HD was also positively associated with increased age $(\mathrm{aHR}=1.011 ; 95 \% \mathrm{CI}=1.005-1.016 ; \mathrm{P}<0.001)$. The association between MM and HD was further analyzed. Table III displays the clinical characteristics of 283 patients 


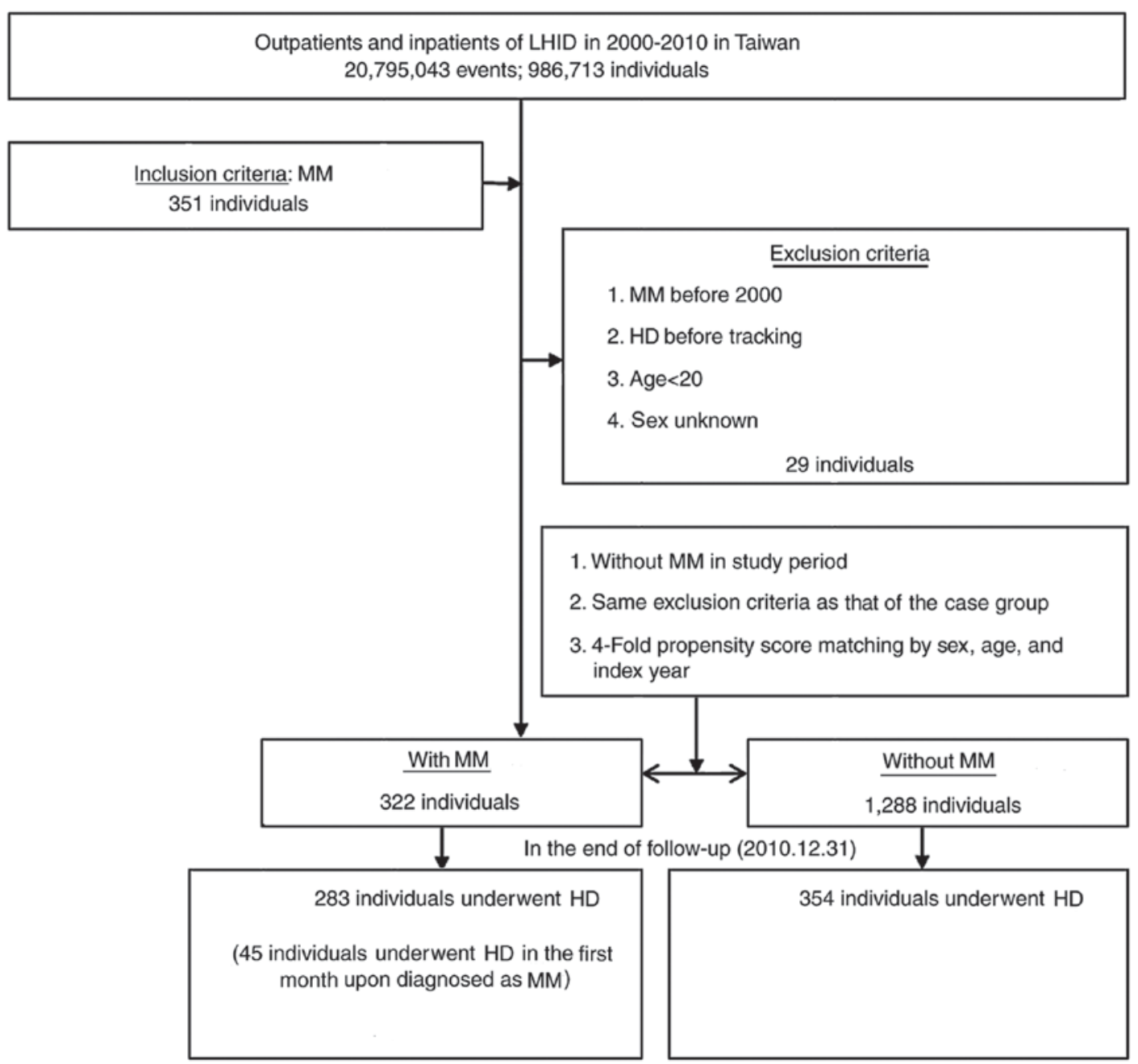

Figure 1. The flowchart of study sample selection from National Health Insurance Research Database in Taiwan. LHID, Longitudinal Health Insurance Database; MM, multiple myeloma; HD, hemodialysis.

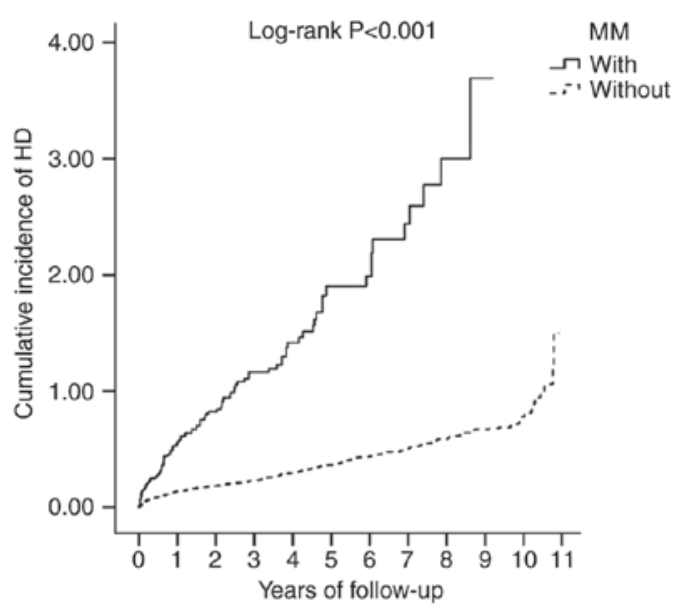

Figure 2. Cumulative incidence of HD treatment among patients with or without MM via Kaplan-Meier curve (log-rank test, $\mathrm{P}<0.001)$. MM, multiple myeloma; HD, hemodialysis.

with MM who received HD and 39 who did not. There was no significant difference between the groups for any characteristic. Following adjusting variables via CCI, there was also no statistical difference between the case and control groups.
The Kaplan-Meier survival curve was used to analyze the cumulative overall survival rate of the study population. It was demonstrated that patients with MM who underwent HD were associated with a significantly reduced survival rate, compared with the control. Additionally, patients with MM who underwent HD demonstrated a significantly decreased survival in the first year following diagnosis in comparision with those without HD (log-rank test, $\mathrm{P}<0.001$; Fig. 3). Furthermore, patients without MM underwent HD were associated with reduced survival in long-term follow-up (Fig. 4). There was sub-analysis of cumulative overall survival between patients treated with HD and anti-myeloma therapy, and HD alone, which revealed no statistical differences between characteristics in these two groups (Table IV). However, the anti-myeloma therapy with HD group were associated with a relatively improved survival rate within the first year following diagnosis (log-rank test $\mathrm{P}=0.116$; Fig. 5). Additionally, another sub-analysis for patients with MM stratified by bone marrow auto-transplantation was conducted. The overall survival curve is presented in Fig. 6. Bone marrow auto-transplantation is a standard management for treatment of MM in $\leq 60$-year-old patients. In long-term follow-up (the maximum was 11 years, and the mean was $4.87 \pm 3.39$ years), the group who underwent 
Table I. Patient characteristics in cohorts with or without MM.

\begin{tabular}{|c|c|c|c|c|c|c|c|}
\hline \multirow[b]{2}{*}{ MM variable } & \multicolumn{2}{|c|}{ Total } & \multicolumn{2}{|c|}{ With } & \multicolumn{2}{|c|}{ Without } & \multirow[b]{2}{*}{ P-value } \\
\hline & $\mathrm{n}$ & $\%$ & $\mathrm{n}$ & $\%$ & $\mathrm{n}$ & $\%$ & \\
\hline Total & 1,610 & & 322 & 20.00 & 1,288 & 80.00 & \\
\hline HD & & & & & & & $<0.001^{\mathrm{a}}$ \\
\hline Without & 973 & 60.43 & 39 & 12.11 & 934 & 72.52 & \\
\hline With & 637 & 39.57 & 283 & 87.89 & 354 & 27.48 & \\
\hline Sex & & & & & & & 0.999 \\
\hline Male & 950 & 59.01 & 190 & 59.01 & 760 & 59.01 & \\
\hline Female & 660 & 40.99 & 132 & 40.99 & 528 & 40.99 & \\
\hline Age (years) & $67.73 \pm 18.15$ & & $68.12 \pm 13.29$ & 6 & $6.39 \pm 18.44$ & & 0.529 \\
\hline $\mathrm{DM}$ & & & & & & & 0.421 \\
\hline Without & 1,422 & 88.32 & 286 & 88.82 & 1,136 & 88.20 & \\
\hline With & 188 & 11.68 & 36 & 11.18 & 152 & 11.80 & \\
\hline Hypertension & & & & & & & 0.042 \\
\hline Without & 1,354 & 84.10 & 260 & 80.75 & 1,094 & 84.94 & \\
\hline With & 256 & 15.90 & 62 & 19.25 & 194 & 15.06 & \\
\hline COPD & & & & & & & 0.020 \\
\hline Without & 1,508 & 93.72 & 310 & 96.27 & 1,198 & 93.08 & \\
\hline With & 101 & 6.28 & 12 & 3.73 & 89 & 6.92 & \\
\hline CAD & & & & & & & 0.261 \\
\hline Without & 1,283 & 79.69 & 252 & 78.26 & 1,031 & 80.05 & \\
\hline With & 327 & 20.31 & 70 & 21.74 & 257 & 19.95 & \\
\hline Stroke & & & & & & & 0.001 \\
\hline Without & 1,524 & 94.66 & 316 & 98.14 & 1,208 & 93.79 & \\
\hline With & 86 & 5.34 & 6 & 1.86 & 80 & 6.21 & \\
\hline CCI_R & $0.56 \pm 1.00$ & & $0.57 \pm 0.94$ & & $0.56 \pm 1.01$ & & 0.940 \\
\hline
\end{tabular}

$\mathrm{n}$, number; ${ }^{\mathrm{P}}<0.001$; Statistical analysis of category variables, $\chi^{2} /$ Fisher exact test; continuous variable, Student's t-test. MM, multiple myeloma; HD, hemodialysis; DM, diabetes mellitus; COPD, chronic obstructive pulmonary disease; CAD, coronary artery disease; CCI_R, Charlson comorbidity index (removed cancers, DM, COPD, CAD and stroke).

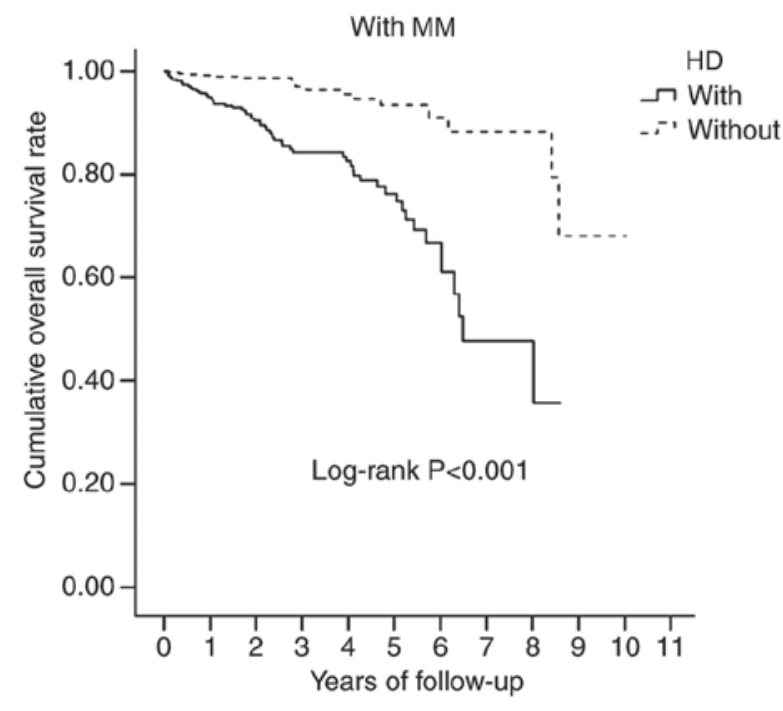

Figure 3. Cumulative overall survival rate of patients with MM with or without HD treatment via Kaplan-Meier curve (log-rank test, $\mathrm{P}<0.001)$. MM. MM, multiple myeloma; HD, hemodialysis.

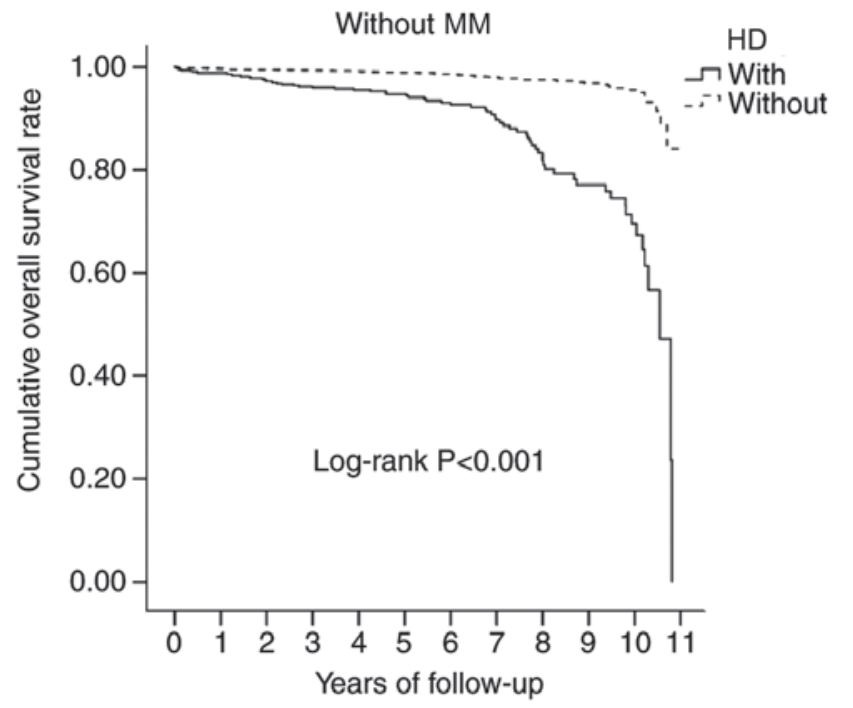

Figure 4. Cumulative overall survival rate of patients without MM with or without HD treatment via Kaplan-Meier curve (log-rank test $\mathrm{P}<0.001)$. MM. multiple myeloma; HD, hemodialysis. 
Table II. Multivariate analysis of factors associated with hemodialysis in cohorts with or without MM.

\begin{tabular}{|c|c|c|c|}
\hline Variable & Adjusted HR & $95 \% \mathrm{CI}$ & P-value \\
\hline \multicolumn{4}{|l|}{ MM } \\
\hline Without & 1.000 & & \\
\hline With & 7.347 & $6.156-8.768$ & $<0.001$ \\
\hline \multicolumn{4}{|l|}{ Sex } \\
\hline Male & 1.135 & $0.964-1.337$ & 0.129 \\
\hline Female & 1.000 & & \\
\hline Age (years) & 1.011 & $1.005-1.016$ & $<0.001$ \\
\hline \multicolumn{4}{|l|}{$\mathrm{DM}$} \\
\hline Without & 1.000 & & \\
\hline With & 1.028 & $0.731-1.179$ & 0.543 \\
\hline \multicolumn{4}{|l|}{ Hypertension } \\
\hline Without & 1.000 & & \\
\hline With & 0.761 & $0.522-1.110$ & 0.156 \\
\hline \multicolumn{4}{|l|}{ COPD } \\
\hline Without & 1.000 & & \\
\hline With & 0.760 & $0.558-1.054$ & 0.071 \\
\hline \multicolumn{4}{|l|}{ CAD } \\
\hline Without & 1.000 & & \\
\hline With & 0.952 & $0.672-1.349$ & 0.784 \\
\hline \multicolumn{4}{|l|}{ Stroke } \\
\hline Without & 1.000 & & \\
\hline With & 1.185 & $0.858-1.638$ & 0.302 \\
\hline CCI_R & 1.145 & $1.060-1.237$ & 0.001 \\
\hline
\end{tabular}

MM, multiple myeloma; HD, hemodialysis; DM, diabetes mellitus; COPD, chronic obstructive pulmonary disease; CAD, coronary artery disease; CCI_R, Charlson comorbidity index (removed cancers, DM, COPD, CAD and stroke).

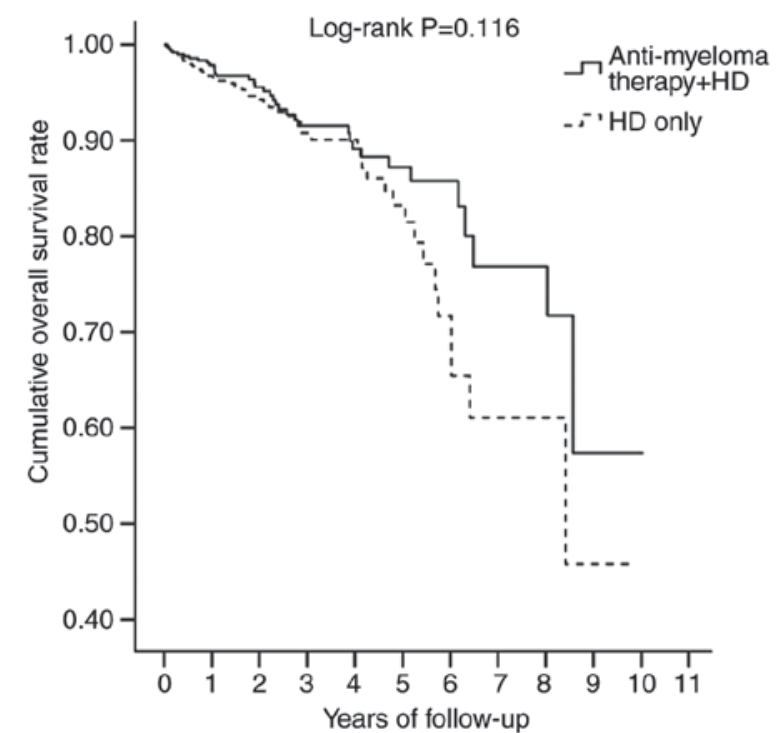

Figure 5. Cumulative overall survival rate of patients with multiple myeloma stratified by anti-myeloma therapy and HD, or HD alone via Kaplan-Meier curve (log-rank test $\mathrm{P}=0.116)$. HD, hemodialysis.

bone marrow auto-transplantation ( $\leq 60$-year-old group) demonstrated a significantly improved overall survival rate

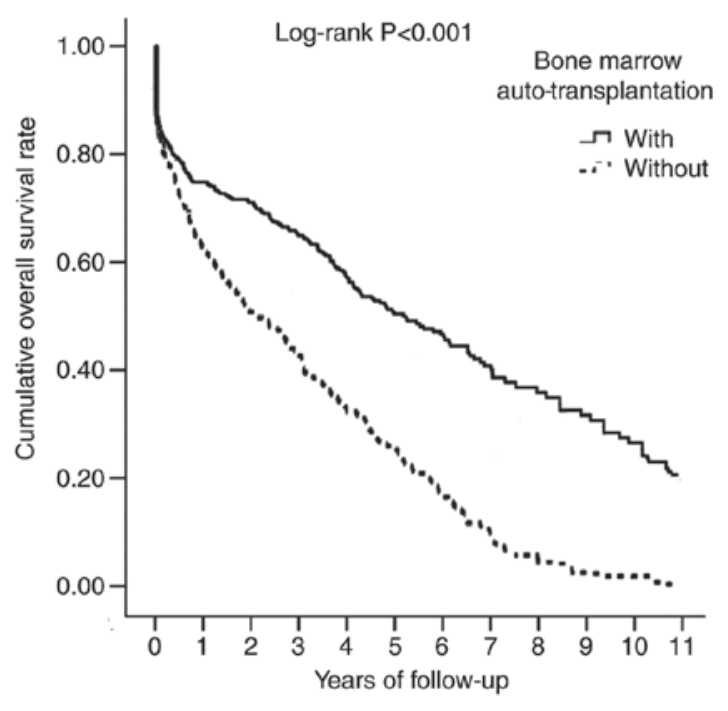

Figure 6. Cumulative overall survival of patients with multiple myeloma who underwent hemodialysis, stratified by bone marrow auto-transplantation via Kaplan-Meier curve (log-rank test $\mathrm{P}<0.001)$.

compared with the group who did not undergo auto-transplantation ( $>60$-year-old group; log-rank test, $\mathrm{P}<0.001$; Fig. 6). 
Table III. Characteristics of multiple myeloma cohorts with or without HD in the past.

\begin{tabular}{|c|c|c|c|c|c|c|c|}
\hline \multirow[b]{2}{*}{ HD variable } & \multicolumn{2}{|c|}{ Total } & \multicolumn{2}{|c|}{ With HD } & \multicolumn{2}{|c|}{ Without HD } & \multirow[b]{2}{*}{ P-value } \\
\hline & $\mathrm{n}$ & $\%$ & $\mathrm{n}$ & $\%$ & $\mathrm{n}$ & $\%$ & \\
\hline Total & 322 & 100 & 283 & 87.89 & 39 & 12.11 & \\
\hline Sex & & & & & & & 0.059 \\
\hline Male & 190 & 59.01 & 172 & 60.78 & 18 & 46.15 & \\
\hline Female & 132 & 40.99 & 111 & 39.22 & 21 & 53.85 & \\
\hline Age (years) ${ }^{\#}$ & $68.12 \pm 13.29^{\mathrm{a}}$ & & $68.14 \pm 13.00^{\mathrm{a}}$ & & $67.97 \pm 15.47^{\mathrm{a}}$ & & 0.542 \\
\hline $\mathrm{DM}$ & & & & & & & 0.125 \\
\hline Without & 286 & 88.82 & 254 & 89.75 & 32 & 82.05 & \\
\hline With & 36 & 11.18 & 29 & 10.25 & 7 & 17.95 & \\
\hline Hypertension & & & & & & & 0.512 \\
\hline Without & 260 & 80.75 & 228 & 80.57 & 32 & 82.05 & \\
\hline With & 62 & 19.25 & 55 & 19.43 & 7 & 17.95 & \\
\hline COPD & & & & & & & 0.522 \\
\hline Without & 317 & 98.45 & 278 & 98.23 & 39 & 100 & \\
\hline With & 5 & 1.55 & 5 & 1.77 & 0 & 0 & \\
\hline CAD & & & & & & & 0.352 \\
\hline Without & 252 & 78.26 & 220 & 77.74 & 32 & 82.05 & \\
\hline With & 70 & 21.74 & 63 & 22.26 & 7 & 17.95 & \\
\hline Stroke & & & & & & & 0.542 \\
\hline Without & 316 & 98.14 & 278 & 98.23 & 38 & 97.44 & \\
\hline With & 6 & 1.86 & 5 & 1.77 & 1 & 2.56 & \\
\hline CCI_R\# & $0.56 \pm 1.00^{\mathrm{a}}$ & & $0.52 \pm 0.83^{\mathrm{a}}$ & & $0.57 \pm 1.02^{\mathrm{a}}$ & & 0.586 \\
\hline
\end{tabular}

$\mathrm{n}$, number; ${ }^{a}$ mean \pm standard deviation; statistical tests, category variable: $\chi^{2} /$ Fisher exact test, continue variable: Student's t-test. DM, diabetes mellitus; HD, hemodialysis; COPD, chronic obstructive pulmonary disease; CAD, coronary artery disease; CCI_R, Charlson comorbidity index (removed cancers, DM, COPD, CAD and stroke).

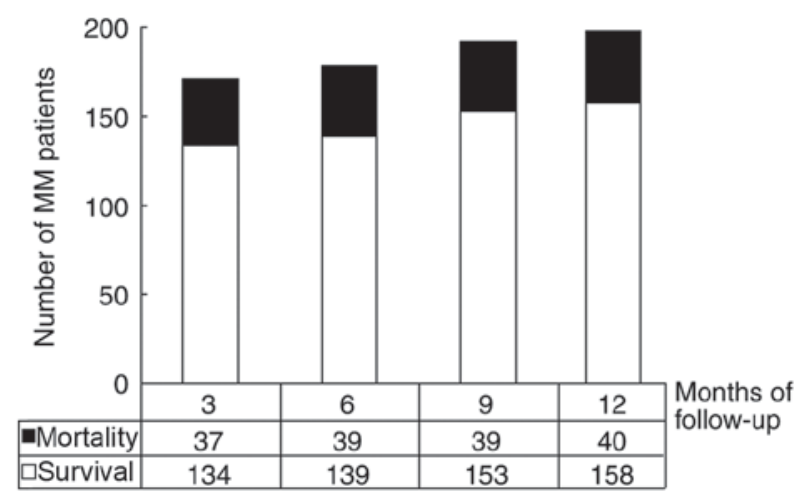

Figure 7. Prognosis of patients with MM who underwent hemodialysis in the first year after diagnosis. MM, multiple myeloma.

A cumulative distribution of prognosis of patients with MM receiving HD in the first year was conducted, and $\leq 198$ (61.49\%) patients received HD in the first year. These cases are distributed into three-month intervals in the bar chart, which revealed that the majority of these patients, $\leq 171$ patients, began HD in the first three months. There were 37 patients in the mortality group and 134 patients in the survival group (Fig. 7). Fisher's exact test no significant difference in

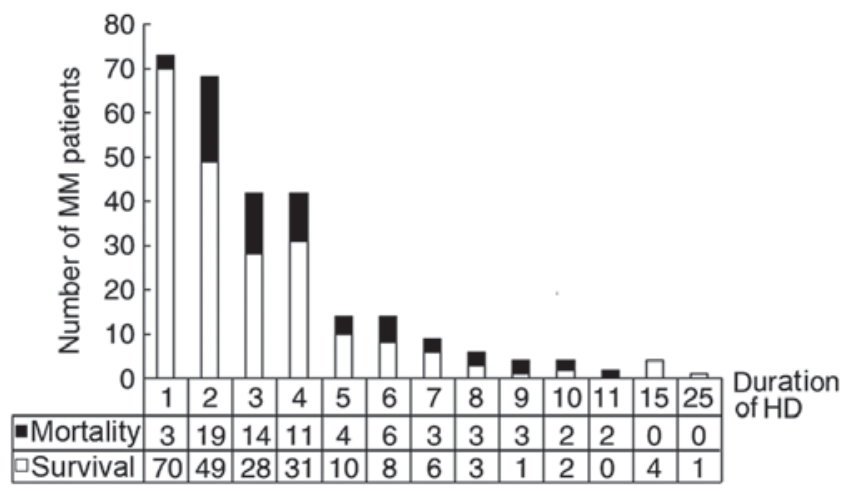

Figure 8. Prognosis of patients with MM and the frequency of HD treatment in the study period. MM, multiple myeloma; HD, hemodialysis.

mortality between the different periods of time $(\mathrm{P}=0.967)$. In addition, the association between prognosis and frequency of HD in patients with MM was investigated. The bar chart in Fig. 8 presents the association between the number of patients with MM and the frequency of patients who underwent HD. The majority of cases underwent $<5$ courses HD $(225 / 283$, $79.5 \%$ ). Cases with a lower frequency of HD were associated with an improved survival status (Fig. 8). Fisher's exact test 
Table IV. Patient characteristics of multiple myeloma cohorts treated with hemodialysis alone and hemodialysis combined anti-myeloma therapy.

\begin{tabular}{|c|c|c|c|c|c|}
\hline \multirow{2}{*}{$\begin{array}{l}\text { Treatment } \\
\text { variable }\end{array}$} & \multicolumn{2}{|c|}{ HD alone } & \multicolumn{2}{|c|}{ HD with anti-myeloma therapy } & \multirow[b]{2}{*}{ P-value } \\
\hline & $\mathrm{n}$ & $\%$ & $\mathrm{n}$ & $\%$ & \\
\hline Total & 165 & 58.30 & 118 & 41.70 & \\
\hline Sex & & & & & 0.389 \\
\hline Male & 104 & 63.03 & 68 & 57.63 & \\
\hline Female & 61 & 36.97 & 50 & 42.37 & \\
\hline Age (years) & $68.15 \pm 12.98^{\mathrm{a}}$ & & $67.97 \pm 13.64^{\mathrm{a}}$ & & 0.910 \\
\hline $\mathrm{DM}$ & & & & & 0.843 \\
\hline Without & 149 & 90.30 & 105 & 88.98 & \\
\hline With & 16 & 9.70 & 13 & 11.02 & \\
\hline Hypertension & & & & & 0.762 \\
\hline Without & 134 & 81.21 & 94 & 79.66 & \\
\hline With & 31 & 18.79 & 24 & 20.34 & \\
\hline COPD & & & & & 0.405 \\
\hline Without & 161 & 97.58 & 117 & 99.15 & \\
\hline With & 4 & 2.42 & 1 & 0.85 & \\
\hline CAD & & & & & 0.405 \\
\hline Without & 130 & 78.79 & 90 & 76.27 & \\
\hline With & 35 & 21.21 & 28 & 23.73 & \\
\hline Stroke & & & & & 0.938 \\
\hline Without & 162 & 98.18 & 116 & 98.31 & \\
\hline With & 3 & 1.82 & 2 & 1.69 & \\
\hline CCI_R & $0.52 \pm 0.82^{\mathrm{a}}$ & & $0.53 \pm 0.86^{\mathrm{a}}$ & & 0.921 \\
\hline
\end{tabular}

demonstrated that there was a statistically significant association between the frequency of patients who underwent HD and mortality $(\mathrm{P}<0.001)$.

In addition to the nationwide population-based cohort study, a single-institute analysis of patients with MM and renal impairment was performed. The patients' profiles are described in Table V. A total of 19 patients with MM and renal impairment consisted of 17 males and 2 females. The patients' mean age was $78.7 \pm 13.4$ years old (range $54-100$ years). The MM stage at diagnosis was in accordance with the International Staging System (ISS) (13). There were two patients with stage 2 disease and 17 patients with stage 3. Renal impairment was analyzed by creatinine and eGFR. A creatinine level $>4 \mathrm{mg} / \mathrm{dl}$ and an eGFR $<30$ were exhibited in all patients. AKIN criteria was used to evaluate the stages of 12 patients with acute kidney injury and if creatinine was rapidly increased within $48 \mathrm{~h}$, and they were diagnosed as AKIN stage 3 disease. A total of 11 patients underwent dialysis, of which 7 patients sustained dialysis-dependence (6 patients received HD and 1 patient received peritoneal dialysis), and 4 patients received emergency HD. Only 1 patient received plasma exchange. At the final follow-up of these cases, 4 patients were alive. A total of 3 patients underwent emergency HD transiently, and subsequently did not rely on HD in the follow-up. A total of 8 patients were heavy chain secretory predominant and 11 patients were light chain secretory predominant. A total of 6 patients underwent renal biopsy, 3 patients had tubulointerstitial nephritis, 1 patient had acute interstitial nephritis, 1 patient had chronic glomerulonephritis and 1 patient had light chain deposition disease.

\section{Discussion}

Renal insufficiency is a common complication observed during the disease course of MM, and it is known as MRKD. The etiology of MRKD-induced acute or chronic kidney injury is caused by excess light chain deposition, particularly the overproduction and filtration of toxic light chains, which contribute to cast formation and tubular obstruction (14). Other predisposing factors include dehydration, hypercalcemia and tumor lysis syndrome, which contribute to acute kidney injury in MRKD (15). However, myeloma cast nephropathy accounts for $\leq 50 \%$ pathogenesis of MRKD, and it is frequently manifested in the acute or subacute phase of kidney injury (16). It has been 


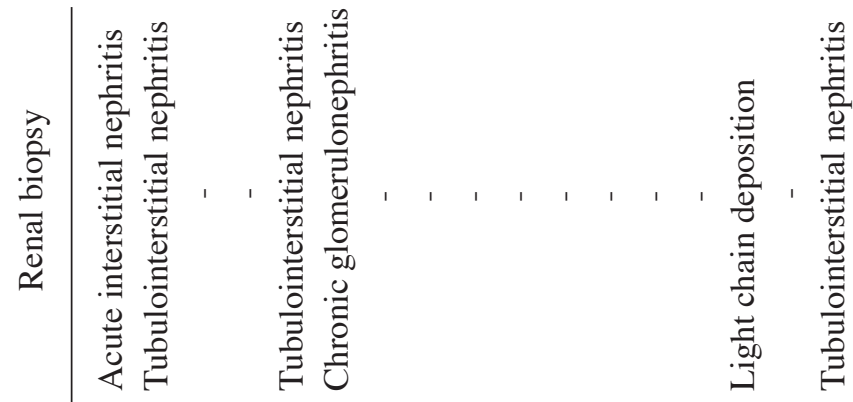

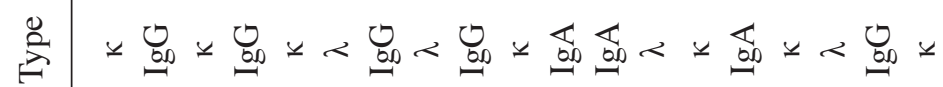

范

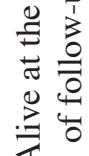

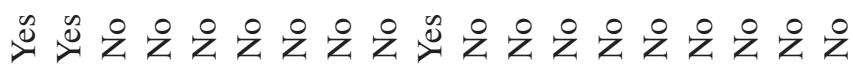

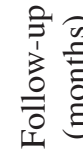

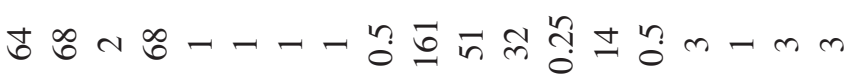

ซี

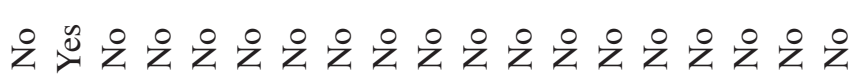

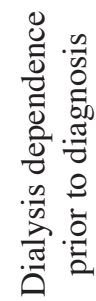

능 主

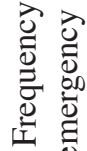

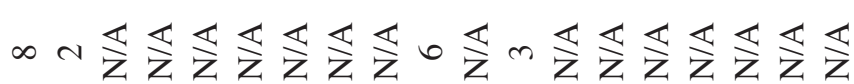

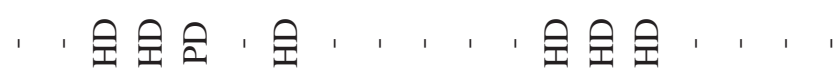
응 홀

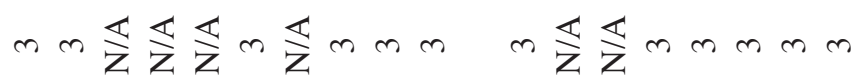

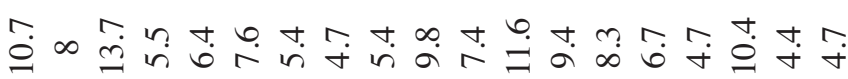

- 
estimated that $50 \%$ patients with MM sustain acute kidney injury or chronic kidney disease during the course of MM (17). However, the majority of patients with MM suffer acute kidney injury rather than chronic kidney disease. It is essential to halt the deterioration of the kidney function before progression to chronic kidney disease or dialysis-dependence (18).

To the best of our knowledge, the present study is the first to investigate the association between HD and survival outcome in patients with MM in Taiwan. Firstly, number of patients requiring HD among those with or without MM was analyzed, and MM was demonstrated to be an independent risk factor for HD (adjusted HR, 7.347). A recent study regarding the nationwide database in Taiwan between 1997 and 2013 containing 7,285 patients with MM and 4,471 patients who underwent $\mathrm{HD}$ (61.37\% of total population) demonstrated there was higher incidence of MRKD in patients with MM than in western countries (19). Furthermore, chronic kidney disease is highly prevalent in Taiwan, and the prevalence of end stage renal disease was 2,451/1,000,000 in 2009. A possible reason for this is that the initial HD modality was notably more accessible in Taiwan, compared with Western countries, and that HD is reimbursed by National health insurance (20). This led to a higher rate of HD $(\leq 89.7 \%)$ in 2009 (20). It was indicated in the present study that overall survival was significantly reduced in patients with MM who underwent HD than those without HD, and a notable decrease in survival in the first year (log-rank test $\mathrm{P}<0.001$; Fig. 3). This may be due to patients being diagnosed with MRKD and acute kidney injury, or other complications, including hypercalcemia, hyperviscosity, coagulopathy and hypogammoglobulinemia. The aforementioned complications may result in an increased mortality rate in patients initially diagnosed with MRKD and receiving HD compared with patients with no other complications. Kumar et al (21) performed a study between 2001 and 2010, and the study population was grouped into two five-year periods at diagnosis (2001-2005 and 2006-2010). Early mortality was lower in the latter group, and was considered to be a result of quickly using advanced agents, including thalidomide, lenalidomide and bortezomib subsequent to diagnosis. There was no statistical difference in cumulative overall survival between patients treated with anti-myeloma therapy and HD, or HD alone. However, it was demonstrated that patients treated with anti-myeloma therapy and HD had a relatively good prognosis compared with those treated with HD alone (Fig. 5). Sub-analysis revealed that patients with MM who underwent bone marrow auto-transplantation ( $\leq 60$-year-old group) demonstrated statistically improved overall survival, compared with patients who did not undergo auto-transplantation ( $>60$-year-old group; log-rank test, $\mathrm{P}<0.001$; Fig. 6).

It was demonstrated that early mortality was significantly associated with various factors, including age of $>70$ years, year of diagnosis, ISS staging and lack of possibility for advanced treatment (21). However, MM is considered a heterogeneous disease, and early mortality may be influenced by other factors, including performance status, serum albumin, serum creatinine, serum calcium, hemoglobin, platelet count and plasma cell percentage in bone marrow (1).

Subsequently, the aim was to determine whether HD may influence the outcome in the first year after diagnosis, and the duration of HD in patients with MM was verified. As for the duration of HD among patients with MM who underwent HD in the first year, the majority of patients received HD transiently following diagnosis with MM with acute kidney injury. The frequency of HD revealed a centralized distribution at $<5$ five courses of HD (Fig. 8), and there was a higher probability of the patient recovering renal function and discontinuing dialysis in patients with MM undergoing HD at the end of follow-up.

Based on this retrospective study, risk stratification of MRKD should be a prioritized consideration following diagnosis of MM, and the determination of HD, including timing, frequencies and duration, may be influenced by risk stratification. Firstly, HD treatment primarily began in the first year following diagnosis of MM with renal insufficiency. The first year of treatment is considered critical in the care of patients with MM with renal insufficiency (22). However, there has been no standardization of renal insufficiency in MM previously. Although CRAB criteria define a creatinine level of $>2 \mathrm{mg} / \mathrm{dl}$ as the standard of renal insufficiency (23), creatinine was considered to be a weak evaluation tool due to it only defining the cut-off value of renal insufficiency in patients with MM, but not early chronic kidney disease or residual renal function in patients with MM. Additionally, creatinine level is affected by decreased muscle mass, age and physiological condition, including external creatinine degradation caused by intestine bacterial growth. When diagnosed with MM, an estimated $30 \%$ patients had a creatinine level $>1.5 \mathrm{mg} / \mathrm{dl}$, and $\leq 13 \%$ required dialysis; therefore, calculation of the eGFR may be a more effective discriminator (7). The International Myeloma Working Group recommends five stages of classification of chronic kidney disease from the Cockcroft-Gault and Modification of Diet in Renal Disease study equations for patients with MRKD $(24,25)$. It was determined that 7 patients from the TSGH were dialysis-dependent prior to diagnosis of MM (Table V). Therefore, it was considered that creatinine, GFR or the five stages of chronic kidney disease were not a sufficient indicator for patients with MM with renal impairment. In particular, acute kidney injury caused by MRKD should be carefully considered. Dimopoulos et al (25) and Mehta et al (26) indicated that risk, injury, failure, loss and end-stage kidney disease (RIFLE) system was not sufficient for assessment of acute kidney injury in MM. Furthermore, the AKIN criteria for evaluation of acute kidney injury were developed and modified by the RIFLE classification. Although the RIFLE classification or AKIN criteria can be applied for classification of renal insufficiency in MM, neither have validated in MRKD. There may be potential for an investigation of risk stratification of renal impairment and subsequent timing of $\mathrm{HD}$ in patients with MM.

Additionally, the retrospective nationwide study revealed there was probability of recovery of renal function and discontinuation of dialysis in patients with MM who underwent HD at the end of follow-up. Furthermore, the TSGH analysis of patients with MM with renal impairment determined that 3 patients had an improved survival outcome and discontinued dialysis. To the best of our knowledge, only one single-center study has discussed renal recovery in patients with MRKD treated with HD (22). Haynes et al (22) postulated that $17 \%$ patients with MRKD receiving dialysis upon diagnosis had subsequently recovered to discontinue dialysis. Among those 
patients with MRKD, 20\% was caused by severe acute kidney injury (17). The goal of renal replacement therapy (RRT) is to allow fluid maintenance and electrolyte correction to achieve renal recovery. In order to consider the frequency, dose and duration it was important to monitor residual renal function, maintain hemodynamic stability, confirm dialysis adequacy and avoid nephrotoxic agents. Prompt timing of RRT for acute kidney injury was considered critical to renal recovery in addition to emergent conditions, including severe acidosis, hyperkalemia, oliguria and fluid overload, the cut-off value of blood nitrogen, serum creatinine or urine output. With regards to the modality of RRT in MRKD in the present study, HD is the most prevalent treatment form in Taiwan, and the preferred approach for the treatment of MRKD with acute kidney injury status (19). The main limitation of HD is failure to remove light chain deposition. Advanced extracorporeal treatments, including plasmapheresis combined with chemotherapy, are advocated for the rapid eradication of nephrotoxic free light chains $(27,28)$. Only one participant in the present study received plasmapheresis due to hyper-viscosity status (Table V); however, only four clinical studies exist, and the effectiveness of renal recovery is controversial (29-32). Plasmapheresis is considered to be more beneficial in patients with MM and hyper-viscosity status (33). Extended daily HD using high cut-off protein-leaking dialyzers is another new treatment modality for cast nephropathy, and it contributes to improved clearance of light chain deposition. Patients treated as such demonstrated improved survival and renal recovery rates compared with patients treated with conventional dialysis. However, prospective studies, including the EuLite (34) and the MYRE (35) trials, may provide more robust evidence of this treatment modality being a superior option (36).

Although renal recovery is associated with a good outcome for patients with MM (37), the definite mechanism remains unclear. In the single-institute analysis of patients with MM with renal impairment, there were 8 patients with heavy-chain type MM, and 11 patients with light-chain type MM. This demonstrated that the serum-free light chains may have a notable impact on the nephrotoxicity of MM: Patients with free light chain levels $>1,000 \mathrm{mg} / \mathrm{l}$ were more prone to renal failure (14). Renal biopsy and hematological work are further indicated for MRKD patients, and a total of 6 patients received renal biopsy at the Nephrology Department of the TSGH. Chow et al (38) emphasized the role of renal biopsy, which is considered a vital management a acute deteriorated renal function and a beneficial method to analyze the cause and reversibility of MRKD. A survey of renal biopsy in 61/211 patients (28.9\%) with myeloma kidney disease revealed the common histological types of myeloma kidney disease, including cast nephropathy, amyloidosis, light chain deposition and tubulointerstitial nephritis. Myeloma cast nephropathy accounts for a notable proportion of monoclonal gammopathies in dialysis, and an increased probability of renal recovery rate was observed in this subtype compared with others (39). As well as being used for investigating the cause of renal failure, renal biopsy can demonstrate the severity of tubulointerstitial nephritis, which is considered a predictor of renal recovery (3). While patients with MRKD sustain albuminuria of $>1 \mathrm{~g} / \mathrm{day}$, it is recommended to perform a differential diagnosis of the possible glomerular lesion by renal biopsy (3); particularly, clinicians would have different therapeutic strategies for histological data. There are variable diagnostic markers of glomerular lesions including focal segmental sclerosis, diffuse mesangial proliferation with sclerosis and minimally changing disease. A previous study suggested that daily protein excretion prior to treatment could be considered a prognostic marker in patients with MM (40).

Despite efforts to control confounding factors, there are a number of limitations in the present study. Firstly, the information obtainable from the NHIRD regarding patient characteristics was lacking in terms of detailed staging of MM, medications used (e.g. NSAIDs or nephrotoxic medication) and treatment modalities (e.g. traditional therapeutic agents or novel agents). Secondly, thorough information regarding the diagnosis of patients with MM with MRKD was not disclosed (e.g. urinalysis, serological laboratory values, imaging and renal biopsy results). Thirdly, despite specialist review, there was potential bias of the NHIRD data due to complicated co-morbidities being missed. Lastly, due to the nature of a retrospective observational study and potential selection bias, further prospective studies may be required.

In conclusion, the present study indicates that MM remains an important risk factor of HD. The main treatment of MRKD includes anti-myeloma therapy and HD, and there was increased mortality in patients with MM undergoing HD treatment in the first year. Analysis of the association between the prognosis and frequency of HD revealed that the majority of patients with MM underwent $<5$ courses of HD. Risk stratification of renal impairment was an important consideration. It was suggested that, in addition to the initiation of advanced chemotherapy, early and prompt HD may be beneficial to patient outcome. Other tools, including renal biopsy, provided notable assistance in the analysis of the cause and reversibility of MRKD. According to a previous studies, the renal response to therapeutic modalities has prognostic value in patients with MRKD, and is associated with improved survival. Therefore, clinicians should aim for the achievement of renal recovery and discontinuation of dialysis.

\section{Acknowledgements}

The authors would like to thank the Cancer Registry Group of Tri-Service General Hospital for clinical data support.

\section{Funding}

No funding was received.

\section{Availability of data and materials}

All data generated or analyzed during this study are included in this published article.

\section{Authors' contributions}

CFC, JHC, WCC and JCL were responsible for study conceptualization, experimentation and software analysis. JHC and $\mathrm{CFC}$ was responsible for manuscript drafting and writing. 
JHC, CFC, WCC, CHC and SNH were responsible for data collection, analysis and interpretation. WCC and $\mathrm{CHC}$ were responsible for formal analysis. JHC, WCC and JCL provided study resources.

\section{Ethics approval and consent to participate}

The investigational protocols were approved by the official peer review committee in the Tri-Service General Hospital and the protocol number was IRB No. 2-105-05-082.

\section{Consent for publication}

Not applicable.

\section{Competing interests}

The authors declare that they have no competing interests.

\section{References}

1. Kyle RA, Gertz MA, Witzig TE, Lust JA, Lacy MQ, Dispenzieri A Fonseca R, Rajkumar SV, Offord JR, Larson DR, et al: Review of 1027 patients with newly diagnosed multiple myeloma. Mayo Clin Proc 78: 21-33, 2003.

2. Kyle RA, Therneau TM, Rajkumar SV, Larson DR, Plevak MF and Melton LJ III: Incidence of multiple myeloma in olmsted county, minnesota. Cancer 101: 2667-2674, 2004.

3. Shi H, Chen Z, Xie J and Chen N: The prevalence and management of multiple myeloma-induced kidney disease in China Kidney Dis 1: 235-240, 2016.

4. Rajkumar SV, Dimopoulos MA, Palumbo A, Blade J, Merlini G, Mateos MV, Kumar S, Hillengass J, Kastritis E, Richardson $\mathrm{P}$, et al: International myeloma working group updated criteria for the diagnosis of multiple myeloma. Lancet Oncol 15: e538-e548, 2014.

5. Landgren $\mathrm{O}$ and Weiss BM: Patterns of monoclonal gammopathy of undetermined significance and multiple myeloma in various ethnic/racial groups: Support for genetic factors in pathogenesis. Leukemia 23: 1691-1697, 2009.

6. Herrera GA and Sanders PW: Paraproteinemic renal diseases that involve the tubulo-interstitium. Contrib Nephrol 153: 105-115, 2007.

7. Dimopoulos MA, Kastritis E, Rosinol L, Bladé J and Ludwig H: Pathogenesis and treatment of renal failure in multiple myeloma. Leukemia 22: 1485-1493, 2008.

8. Tsakiris DJ, Stel VS, Finne P, Fraser E, Heaf J, de Meester J, Schmaldienst S, Dekker F, Verrina E and Jager KJ: Incidence and outcome of patients starting renal replacement therapy for end-stage renal disease due to multiple myeloma or light-chain deposit disease: An ERA-EDTA Registry study. Nephrol Dial Transplant 25: 1200-1206, 2010.

9. Chen YC, Yeh HY, Wu JC, Haschler I, Chen TJ and Wetter T: Taiwan's National health insurance research database: Administrative health care database as study object in bibliometrics. Scientometrics 86: 365-380, 2011.

10. Slee VN: The International classification of diseases: Ninth revision (ICD-9). Ann Intern Med 88: 424-426, 1978.

11. Lopes JA, Fernandes P, Jorge S, Gonçalves S, Alvarez A, Costa e Silva Z, França C and Prata MM: Acute kidney injury in intensive care unit patients: A comparison between the RIFLE and the acute kidney injury network classifications. Crit Care 12: R110, 2008.

12. Deyo RA, Cherkin DC and Ciol MA: Adapting a clinical comorbidity index for use with ICD-9-CM administrative databases. J Clin Epidemiol 45: 613-619, 1992.

13. Snozek CL, Katzmann JA, Kyle RA, Dispenzieri A, Larson DR, Therneau TM, Melton LJ III, Kumar S, Greipp PR, Clark RJ and Rajkumar SV: Prognostic value of the serum free light chain ratio in newly diagnosed myeloma: Proposed incorporation into the international staging system. Leukemia 22: 1933-1937, 2008.
14. Hutchison CA, Batuman V, Behrens J, Bridoux F, Sirac C, Dispenzieri A, Herrera GA, Lachmann H and Sanders PW; International Kidney and Monoclonal Gammopathy Research Group: The pathogenesis and diagnosis of acute kidney injury in multiple myeloma. Nat Rev Nephrol 8: 43-51, 2011.

15. Sakhuja V, Jha V, Varma S, Joshi K, Gupta KL, Sud K and Kohli HS: Renal involvement in multiple myeloma: A 10-year study. Ren Fail 22: 465-477, 2000.

16. Walther C, Podoll AS and Finkel KW: Treatment of acute kidney injury with cast nephropathy. Clin Nephrol 82: 1-6, 2014.

17. Yadav P, Cook M and Cockwell P: Current trends of renal impairment in multiple myeloma. Kidney Dis 1: 241-257, 2016.

18. Knudsen LM, Hjorth M and Hippe E: Renal failure in multiple myeloma: Reversibility and impact on the prognosis. Nordic Myeloma Study Group. Eur J Haematol 65: 175-181, 2000.

19. Chen JH, Chung CH, Wang YC, Hsu SN, Huang WY and Chien WC: Prevalence and mortality-related factors of multiple myeloma in Taiwan. PLoS One 11: e0167227, 2016.

20. Health NIo: US Renal Data System, USRDS 2010 Annual data report: Atlas of chronic kidney disease and end-stage renal disease in the United States. National Institute of Diabetes and Digestive and Kidney Diseases, 2010.

21. Kumar SK, Dispenzieri A, Lacy MQ, Gertz MA, Buadi FK, Pandey S, Kapoor P, Dingli D, Hayman SR, Leung N, et al: Continued improvement in survival in multiple myeloma: Changes in early mortality and outcomes in older patients. Leukemia 28: 1122-1128, 2014.

22. Haynes RJ, Read S, Collins GP, Darby SC and Winearls CG: Presentation and survival of patients with severe acute kidney injury and multiple myeloma: 20-year experience from a single centre. Nephrol Dial Transplantat 25: 419-426, 2010.

23. International Myeloma Working Group: Criteria for the classification of monoclonal gammopathies, multiple myeloma and related disorders: A report of the International Myeloma Working Group. Br J Haematol 121: 749-757, 2003.

24. Levey AS, Eckardt KU, Tsukamoto Y, Levin A, Coresh J, Rossert J, De Zeeuw D, Hostetter TH, Lameire N and Eknoyan G: Definition and classification of chronic kidney disease: A position statement from kidney disease: Improving global outcomes (KDIGO). Kidney Int 67: 2089-2100, 2005.

25. Dimopoulos MA, Terpos E, Chanan-Khan A, Leung N, Ludwig H, Jagannath S, Niesvizky R, Giralt S, Fermand JP, Bladé J, et al: Renal impairment in patients with multiple myeloma: A consensus statement on behalf of the International Myeloma Working Group. J Clin Oncol 28: 4976-4984, 2010.

26. Mehta RL, Kellum JA, Shah SV, Molitoris BA, Ronco C, Warnock DG and Levin A; Acute Kidney InjuryNetwork: Acute kidney injury network: Report of an initiative to improve outcomes in acute kidney injury. Crit Care 11: R31, 2007.

27. Hutchison CA, Bradwell AR, Cook M, Basnayake K, Basu S, Harding S, Hattersley J, Evans ND, Chappel MJ, Sampson P, et al: Treatment of acute renal failure secondary to multiple myeloma with chemotherapy and extended high cut-off hemodialysis. Clin J Am Soc Nephrol 4: 745-754, 2009.

28. Hutchison CA, Cockwell P, Stringer S, Bradwell A, Cook M, Gertz MA, Dispenzieri A, Winters JL, Kumar S, Rajkumar SV, et al: Early reduction of serum-free light chains associates with renal recovery in myeloma kidney. J Am Soc Nephrol 22: 1129-1136, 2011.

29. Zucchelli P, Pasquali S, Cagnoli L and Ferrari G: Controlled plasma exchange trial in acute renal failure due to multiple myeloma. Kidney Int 33: 1175-1180, 1988.

30. Johnson WJ, Kyle RA, Pineda AA, O'Brien PC and Holley KE: Treatment of renal failure associated with multiple myeloma. Plasmapheresis, hemodialysis, and chemotherapy. Arch Intern Med 150: 863-869, 1990.

31. Clark WF, Stewart AK, Rock GA, Sternbach M, Sutton DM, Barrett BJ, Heidenheim AP, Garg AX, Churchill DN and Canadian Apheresis Group: Plasma exchange when myeloma presents as acute renal failure: A randomized, controlled trial. Ann Intern Med 143: 777-784, 2005.

32. Gaskin G: MyEloma Renal Impairment Trial: Adjunctive plasma exchange in patients with newly diagnosed multiple myeloma and acute renal failure (MERIT): NCT00416897; ISRCTN37161699.

33. Madore F: Plasmapheresis in cast nephropathy: Yes or no? Curr Opin Nephrol Hypertens 24: 177-182, 2015.

34. Hutchison CA, Cook M, Heyne N, Weisel K, Billingham L, Bradwell A and Cockwell P: European trial of free light chain removal by extended haemodialysis in cast nephropathy (EuLITE): A randomised control trial. Trials 9: 55, 2008. 
35. Bridoux F and Fermand JP: Optimizing treatment strategies in myeloma cast nephropathy: Rationale for a randomized prospective trial. Adv Chronic Kidney Dis 19: 333-341, 2012.

36. Curti A, Schwarz A, Trachsler J, Tomonaga Y and Ambühl PM: Therapeutic efficacy and cost effectiveness of high cut-off dialyzers compared to conventional dialysis in patients with cast nephropathy. PLoS One 11: e0159942, 2016.

37. Laforet M, Jourde-Chiche N, Haddad F, Sallee M, Stoppa AM, Brunet P, Dussol B, Burtey S and Gondouin B: Evolution in the treatment of multiple myeloma and impact on dialysis independence: Data from a French cohort from 1999 to 2014. Blood Cancer J 6: e409, 2016.
38. Chow CC, Mo KL, Chan CK, Lo HK, Wong KS and Chan JC: Renal Impairment in patients with multiple myeloma. Hong Kong Med J 9: 78-82, 2003.

39. Decourt A, Gondouin B, Delaroziere JC, Brunet P, Sallée M, Burtey S, Dussol B, Ivanov V, Costello R, Couchoud C and Jourde-Chiche N: Trends in survival and renal recovery in patients with multiple myeloma or light-chain amyloidosis on chronic dialysis. Clin J Am Soc Nephrol 11: 431-441, 2016.

40. Chen JH, Hsu SN, Huang TC, Wu YY, Lin C, Chang PY, Chen YC and Ho CL: Prognostic significance of initial serum albumin and 24 hour daily protein excretion before treatment in multiple myeloma. PLoS One 10: e0128905, 2015. 